This item was submitted to Loughborough's Research Repository by the author.

Items in Figshare are protected by copyright, with all rights reserved, unless otherwise indicated.

\title{
Virtual task simulation for inclusive design
}

PLEASE CITE THE PUBLISHED VERSION

PUBLISHER

(C) Springer Verlag

VERSION

AM (Accepted Manuscript)

LICENCE

CC BY-NC-ND 4.0

\section{REPOSITORY RECORD}

Marshall, Russell, Keith Case, Steve Summerskill, Ruth Sims, Diane E. Gyi, and Peter M. Davis. 2019. "Virtual Task Simulation for Inclusive Design". figshare. https://hdl.handle.net/2134/5994. 
This item was submitted to Loughborough's Institutional Repository (https://dspace.lboro.ac.uk/) by the author and is made available under the following Creative Commons Licence conditions.

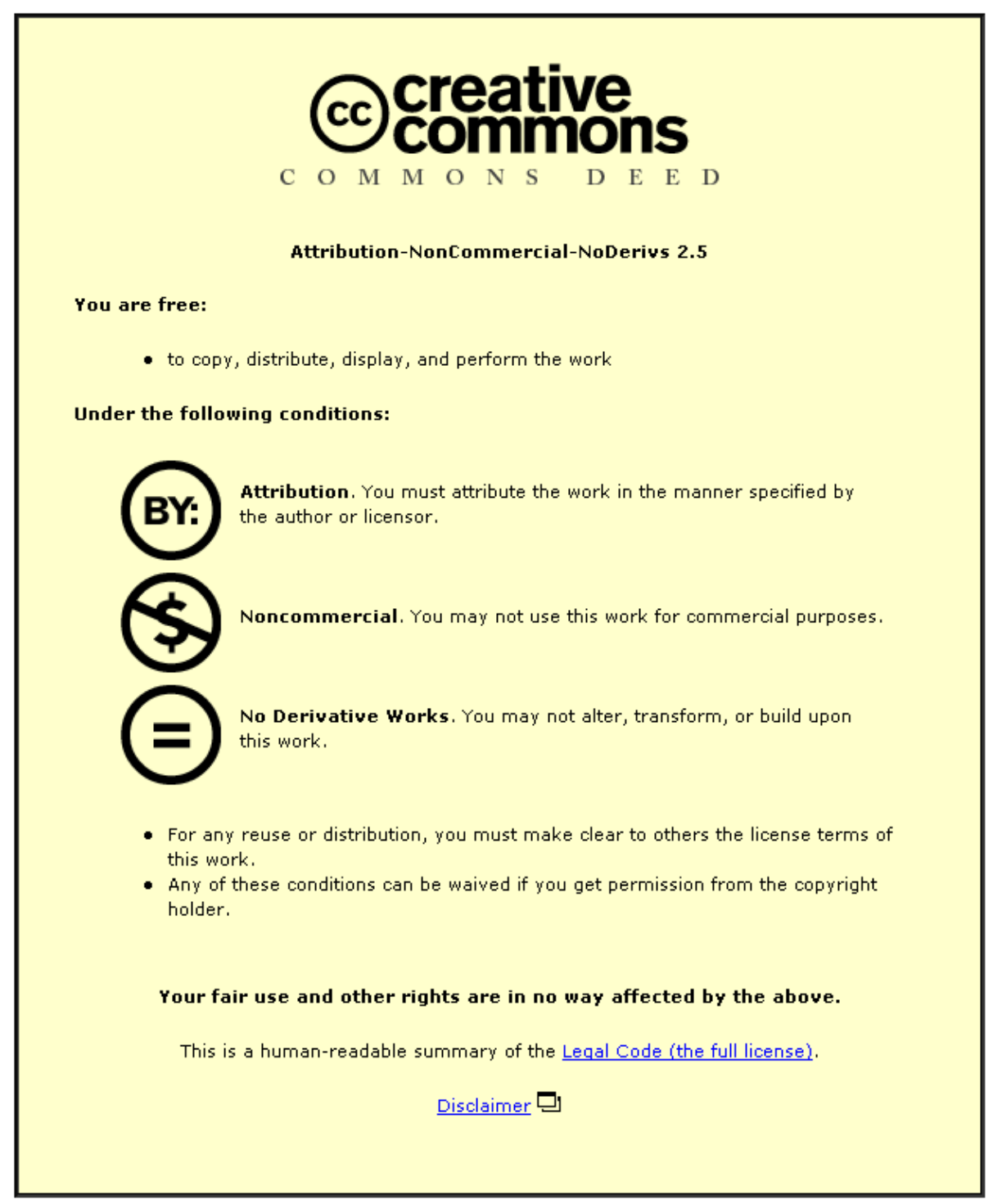

For the full text of this licence, please go to: http://creativecommons.org/licenses/by-nc-nd/2.5/ 


\title{
Virtual Task Simulation for Inclusive Design.
}

\author{
Russell Marshall ${ }^{1}$, Keith Case $^{2}$, Steve Summerskill ${ }^{1}$, Ruth Sims ${ }^{1}$, Diane Gyi ${ }^{3}$ and \\ Peter Davis $^{1}$ \\ ${ }^{1}$ Department of Design and Technology, ${ }^{2}$ Mechanical and Manufacturing Engineering, \\ ${ }^{3}$ Department of Human Sciences, Loughborough University, LE11 3TU, UK \\ \{r.marshall, k.case, s.j.summerskill2, r.sims, d.e.gyi, p.m.davis\}@lboro.ac.uk
}

\begin{abstract}
Human modelling tools provide a means to perform virtual task evaluations upon designs within the computer environment. The ability to evaluate the accommodation of a design early on in the design process before physical prototypes can be built has many advantages. These advantages are particularly relevant in supporting people in attempting to design products that are inclusive and accessible. HADRIAN is a new tool developed to provide accessible, and applicable data on people with a broad range of size, age, and ability together with a means of optimising virtual task evaluations. This paper describes the use of HADRIAN in performing a task evaluation, focusing on the underlying methodology that aims to achieve a virtual simulation that mimics a real world user trial.
\end{abstract}

Keywords: Human modelling, simulation, inclusive design, ergonomics

\section{Introduction}

Human modelling tools provide a highly visual, interactive and timely means to address physical ergonomics problems of posture, fit, reach and vision during product design. Users of different sizes and shapes can be manipulated to simulate interactions with a computer model of an existing or proposed design. However, whilst such tools can be used effectively and efficiently to determine how successful a design may be in accommodating its users, they are not without their shortcomings. Research conducted by Loughborough University in the UK and funded by the Engineering and Physical Research Council (EPSRC) has been developing a means to address two significant issues associated with human modelling. These issues are the relevance, accessibility and applicability of the data used to drive the human model, and a means to simplify the often complex task of manipulating the human model into a representative posture during a product assessment.

The first phase of the research was conducted under the Design for All element of the Extending Quality Life (EQUAL) programme of the EPSRC. Its aim was to address the data and simplification of human modelling use, issues with a particular focus on 'design for all' or inclusive design. More recently the research has progressed expanding its scope and targeting a specific application, that of transport. This second phase is being conducted as part of the EPSRC's Sustainable Urban 
Environment Programme (SUE) and is called Accessibility and User Needs in Transport (AUNT-SUE) [1].

Human modelling's benefits are particularly applicable to inclusive design problems where the variety of human capability needs to be fully understood if a truly accessible solution is to be realised. However, existing data with which human models are currently constructed has many limitations. A widely used data source in the UK is Adultdata, published by the Department of Trade and Industry [2]. The data span 266 physical body measurements for multiple nationalities. However, not all measures are available for all nationalities, so for example, it is possible to obtain a stature measurement for the German population but there is no German data for arm length. In addition most of the data was collected many years ago. Adultdata was published in 1998, but the sources of data within it range from 1969 to 1998. Investigating further highlights other issues such as the fact that the Chinese data was actually collected from Singapore and Hong Kong.

Other databases such as SizeUK [3] and CAESAR [4] are significantly more recent and their size and sampling strategies make them much more representative of their respective populations. However, they are expensive and often beyond the reach of many designers.

For designers wishing to design for all there are more fundamental concerns. Adultdata and CAESAR do not have data on people who are older than 65 years although SizeUK does have people up to the age of 91 and Older Adultdata (one of the Adultdata series together with Childdata) have data from people over 90 for some nationalities. This is a common limitation for anthropometric databases and thus changes to body size and shape as people age are not reflected in the data. The lack of data from people with limited mobility is also a fundamental issue. The effects of common impairments are rarely reflected in anthropometric data and when they are they tend to be from samples of limited size or with other limiting factors [5] [6].

To address these issues associated with appropriate data a new database has been developed that captures a significant amount of data on 102 people, the majority of whom are older and have some form of disability [7]. These data are stored as a set based around the individual from which they were captured. The data incorporate an image of the individual together with background data and an extensive set of anthropometric, joint mobility, capability and behavioural data all of which can be explored by a designer or ergonomist wishing to examine their user population in greater detail. Storing the data as an individual is an attempt to foster empathy with the end user, where the data could be seen to be representative of a person as opposed to a statistical table or numbers. This approach also directly supports the use of human modelling. An individual can easily be recreated when stored in this manner, thus addressing a fundamental issue with existing data, where a human model would have to be reconstructed from a highly decomposed set of measures from which the variability of real people is difficult to capture [8].

In addition to the database, a tool has also been developed to assist designers and ergonomists in their efforts to design for all through the provision of a means to conduct an ergonomics assessment of a product in the virtual environment by making use of human modelling. Acknowledging the skill and expertise required to accurately capture and represent realistic postures in a human modelling system, the tool has been developed to encourage the user to describe the task they wish to 
perform as opposed to driving the human model. The user then focuses their efforts in describing a task allowing the tool to perform the complex manipulation of the human model in an attempt to perform that task. The tool provides a summary of how successful the people in the database were in performing the task and allows the user to explore difficulties individuals experienced and to try out potential solutions. In effect, the tool acts as means of conducting virtual fitting trials with the individuals in the database forming a readily accessible virtual user group.

\section{HADRIAN}

The combined database and task analysis tool is known as HADRIAN (Human Anthropometric Data Requirements Investigation and ANalysis) [9]. Whilst HADRIAN provides data and a means to perform a task analysis it works together with an existing human modelling tool: SAMMIE [10]. This paper will now focus on the task analysis component of the HADRIAN system, describing its use, the underlying methodology that enables the virtual task simulation to take place, and its relationship with SAMMIE.

\subsection{Basic Approach}

To define a task HADRIAN takes the approach that a task is essentially a dynamic process consisting of a series of smaller elements that combine to achieve a particular goal. HADRIAN then essentially looks to determine key-frames, or static snapshots, of the task at the moment these smaller elements occur. To simplify the process SAMMIE is utilised for its functionality to model the elements of these static snapshots, namely: a posture for the human model, a target object, and an environment. Therefore, the main requirement for the task analysis tool is to support the use of the data in the database in determining a suitable and realistic posture for the key-frame. SAMMIE also contains some tools to aid in the process of determining a posture for task related elements such as reach in addition to a number of standardised postures. Though these tools are not a complete solution they provide a starting point which is manipulated by HADRIAN based upon the data stored in the database to achieve a more realistic posture and thus potentially more realistic outcome for the task analysis.

\subsection{Task Elements}

The key frames or static snapshots identified previously are referred to as task elements in HADRIAN. Tasks are therefore essentially combinations of task elements each governed by an environment consisting of the CAD model to be assessed, a set of parameters associated with the task, and some understanding of the sequence of task elements. Together these components influence the posture of the human model and effectively determine if the human model can achieve a posture that would result in a successful completion of the task element, and thus the task. 
The synthesis of a posture in response to the demands of a task element is performed through a number of mechanisms that may be manipulated to form the posture. These mechanisms include:

- Vision: position of head, neck and eyes in order to successfully view the target

- Reach: position of hand, forearm and upper arm (or foot, calf and thigh) in order to reach the target

- Attitude: position and orientation of other body elements to aid in vision and reach mechanisms

- Posture: the starting posture's influence as an initial component of the overall posture

- Location: position and orientation of the human as a whole

- Sequence: a manipulation of the vision, reach, attitude, and location posture elements taking into account the previous and future postural key-frames. Sequence may also take account of loading / strength factors and their influence on the overall posture.

As a further influence upon the posture adopted at each task element key-frame a number of additional task parameters have been defined including: the preferred hand for the task (if required); the desired or required grip type; the part of the body with which to reach; how long (time) the task element is to be performed for; the number of times the task element is to be performed; the task elements that are dependent on the current task element being completed; the important of the task element to the overall task; and the success parameters for a specific task mechanism e.g. view distance.

\subsection{Task Description}

This syntax defined in the task elements provides a set of influences upon the task. However not all of these influences need concern the user, or person specifying the task. Indeed a particular feature in the development of HADRIAN was how to balance the natural language way of describing a task that a person would normally use, with the much more specific and detailed description that a computer based tool would need in order to interpret what was trying to be achieved. Figure 1 illustrates how three different levels of describing a task may be defined.

The Natural language level is the most user friendly and accessible but is far too vague to drive an automated analysis. The user level is an intermediate level where the user is able to build a task through the use of accessible commands together with a target for that command. The system level is how the system interprets the specified command and the mechanisms it employs in order to respond. Though the syntax at user and system level are similar, it is the approach of the system in interpreting the commands and specifically the way in which an unsuccessful task element attempt is managed that defines the system's response. 


\begin{tabular}{|c|c|c|}
\hline $\begin{array}{l}\text { Natural } \\
\text { language }\end{array}$ & User level & System level \\
\hline \multirow{4}{*}{$\begin{array}{l}\text { Put the } \\
\text { oven on. }\end{array}$} & $\begin{array}{l}\text { View oven control } \\
\text { (identify appropriate } \\
\text { control) }\end{array}$ & 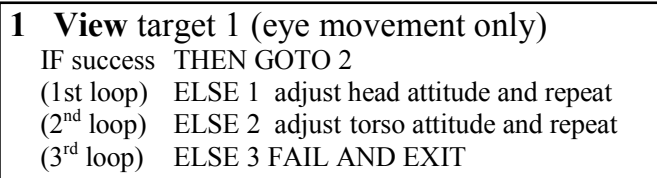 \\
\hline & $\begin{array}{l}\text { Reach oven } \\
\text { control }\end{array}$ & 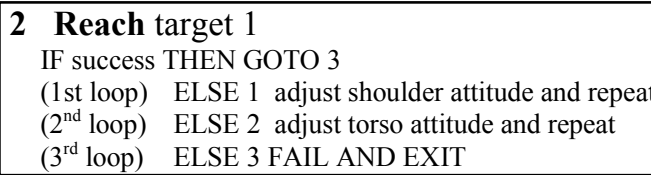 \\
\hline & $\begin{array}{l}\text { Operate oven } \\
\text { control }\end{array}$ & $\begin{array}{l}3 \text { Analyse capability for this posture } \\
\text { IF ok THEN GOTO } 4 \\
\text { (1st loop) } \\
\left(2^{\text {nd }} \text { loop }\right) \\
\text { ELSE } 1 \text { adjust torso attitude and GOTO } 2 \\
\end{array}$ \\
\hline & $\begin{array}{l}\text { View oven } \\
\text { control } \\
\text { (check the correct } \\
\text { setting) }\end{array}$ & 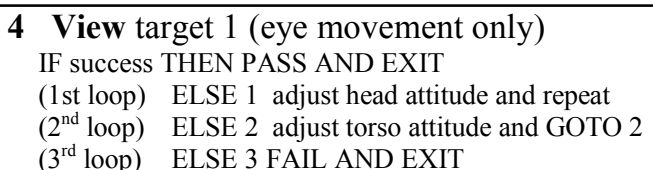 \\
\hline
\end{tabular}

Fig. 1. An example hierarchy of task description.

In HADRIAN the user level is employed to describe task elements and ultimately a task. The system provides a set of commands for the user to select as appropriate and a facility to select the appropriate target for each command.

The details of all HADRIAN task commands is beyond the scope of this paper, but a single HADRIAN command example (reach) is shown below:

Syntax

REACH target [parameters]

Where: target is a named and existing object in the environment; parameters is a comma-separated list of:

- HND = hand is a character value ( $\mathrm{n}=$ nearest, $\mathrm{r}=$ right, $\mathrm{l}=\mathrm{left}, \mathrm{b}=$ both) representing the appropriate hand for the task (unspecified = default hand).

- or ANA = anatomical reference is a character value representing the appropriate part of the body for the task $(\mathrm{h}=$ hand, $\mathrm{f}=$ foot)

- or GRP = grip is a character value representing the grip type (unspecified $=$ default, $\mathrm{o}=$ none, $\mathrm{f}=$ fingertip, $\mathrm{t}=$ thumbtip, $\mathrm{p}=$ palm)

- or DUR = duration is an integer value representing the number of subsequent task elements for which to maintain the reach posture (unspecified $=$ whole of task, $0=$ this task element only).

- or GTE = gate is a comma-separated list of task identifiers.

- or IMP = importance is an integer value (1-10) representing the task-based importance of this task element. 
Example

reach credit_card $(\mathrm{HND}=r, \mathrm{DUR}=0, \mathrm{GRP}=\mathrm{t}, \mathrm{IMP}=10)$

Identifies that an object named credit_card is to be reached using a right-handed thumbtip grip, that the reach posture will be maintained for this task element only, that no other task elements can be completed until the credit_card has been reached, and that this task element has the maximum overall importance.

The pedantic nature of the commands that are utilised within HADRIAN is a deliberate attempt to provide a structured control language that may be used to drive a virtual task simulation irrespective of the particular implementation within HADRIAN. It is recognised that this level of detail is not particularly user friendly and thus the implementation of the HADRIAN interface largely shields the user from this format. However, the command structure does provide a common interface to the task analysis capability within SAMMIE and thus a way for other implementations to be developed, or the functionality accessed without the direct need for HADRIAN.

\subsection{System Strategies}

Once a task has been defined by the user the analysis is implemented or 'run'. The implementation of task commands within the system involves a combination of: the interpretation of task commands into the existing capabilities of SAMMIE, a set of adaptation processes for dealing with common task situations and eventualities, and the management of task element interaction through the task framework. An example of the interpretation of a task command into SAMMIE is shown below

\subsubsection{Command Interpretation}

LOOK command

The LOOK statement initiates a single SAMMIE view command aimed at the specified target. In addition, the distance between the eye-point and the target is determined and compared to the default view distance or the command parameter: value.

\section{REACH command}

The REACH statement initiates a single SAMMIE reach command aimed at the specified target. The reach can be performed using the default human model hand (e.g. left hand for left handed people) or the command parameter: hand with the default human model grip or the command parameter: grip. For two handed grip objects, two reach commands are issued, one for each hand. Alternatively, the REACH command can be initiated using the command parameter: anatomical reference for specific hand reaches (forced to use right hand even if human is left handed), or reaches with the foot. 


\subsubsection{Adaptation Processes}

As we have seen each task command within HADRIAN contains a number of parameters. However, not all of these parameters are mandatory. For optional parameters, HADRIAN has been developed to be adaptable in its response to the command. The first situation requiring a flexible response by the system is in the event of a missing piece of key information such as which hand to use for a reach operation. In such cases the system interrogates the data it has available to it. The first is the data on the human model taken from the HADRIAN database. Relevant data in this example may include handedness, or anthropometry and joint mobility for the arms. The second is the location and orientation of the human with respect to the target for the task command. This data will inform how the system responds and ultimately decides which hand to use for the task.

The most significant area of system adaptation to the task analysis is in the event of a task element failure. In these instances the system follows a basic core process:

1. If the failure is absolute, such as a strength check failure then we move on to point 7. If the failure is postural we continue.

2. Determine relative positioning of the target and the human model. The area around the human model is partitioned into 8 zones for reach (Figure 2a): 2 vertical zones (first digit [0-]) and 4 horizontal zones (second digit [-0]); or 5 zones for vision (Figure 2b). The appropriate zone is determined for the target.

3. For targets in zones 14,24 or 34 the human model is turned through $180^{\circ}$ and the task element repeated. For opposite zones, such as 13/33 for right limb reach or $12 / 32$ for left limb reach, the nearest limb will be used (if possible) or the human model will be turned $90^{\circ}$. For low zones, such as 31 for vision, the human model will crouch or kneel.

4. Measure distance between the target and the key human model reference (e.g. eyepoint for vision, shoulder for arm reach). If out of reach by a large margin then we move to point 6 . If not, continue.

5. Based upon reference-target distance one or more of the following will be applied:

- The head /neck is rotated such that the head is facing the target

- The head /neck is rotated and extended / flexed such that the eye-point to target distance equals the desired parameter value

- The torso is rotated and flexed such that the eye-point to target distance equals the desired parameter value

- The reference to target vector is calculated and the shoulder 'pointed' along that vector to achieve a successful upper-limb reach

- The torso is rotated and flexed in the direction of the target to achieve a successful upper-limb reach.

If there is still a failure we move on to point 7 .

6. If the reference to target distance is greater than those accommodated by a posture change one or more of the following will be applied:

- The human model is turned to face the target.

- The human model is moved closer to the target.

If there is still a failure we move on to point 7 .

7. In the event of absolute failure, a failure is flagged for the results and the next task element is addressed. 


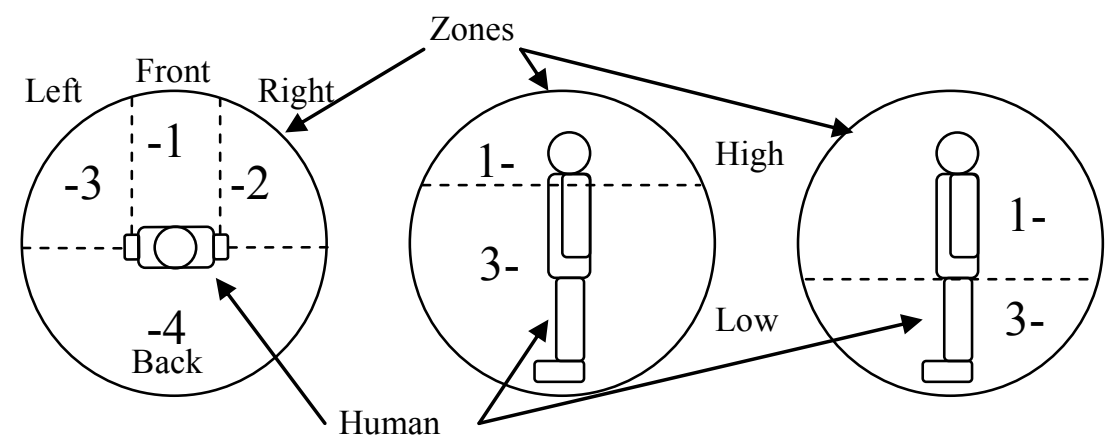

Fig. 2a. Reach orientation zones.

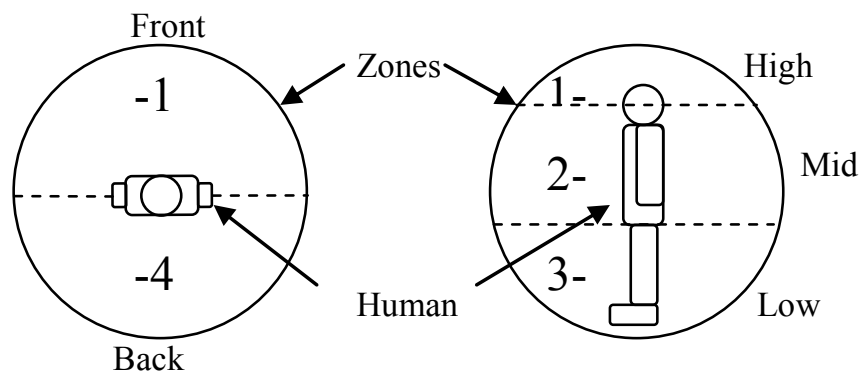

Fig. 2b. View orientation zones.

\subsubsection{Task Framework}

The task framework is an attempt to adjust the human model's approach to each task element based on consequent and subsequent task elements. For the system, this requires information on the 'likely' location, orientation and posture of the human model and any specific details such as the hand to be used and any objects to be interacted with for each task element.

The framework is based on information given during the process of task definition. From the task description the framework specifies the number of task elements, which of those elements are dependent on each other and in what way. The framework also identifies all of the objects that are targets of the task elements and creates a map of the main areas of activity.

The system process to develop the framework consists of the following steps:

1. From task definition determine number of task elements.

2. Scan the task definition for those task elements that are linked through the gate parameter and build a framework map.

3. Scan the task definition for the duration parameter and add this to the framework map.

4. Identify all target objects and collect their locations from the model. Analyse their layout and determine the location and orientation for the human model (Figure 3): 
- Overlay a $1 \mathrm{~m}$ by $1 \mathrm{~m}$ grid on the environment

- Identify the 'working' grid areas (i.e. those that contain an interaction)

- Weight the working areas according to the number of task elements per grid and duration

- Determine human model locations and orientations for task elements:

- Check weighting and adjacency of each grid. For adjacent and equally weighted grids adopt a mean location and orientation. For adjacent and nonequally weighted grids bias the location and orientation towards the greater weighting. For non-adjacent grids start a new location and orientation.

5. Collect target interaction specifics (e.g. hand to use) from the task element targets. Refine the framework map accordingly.

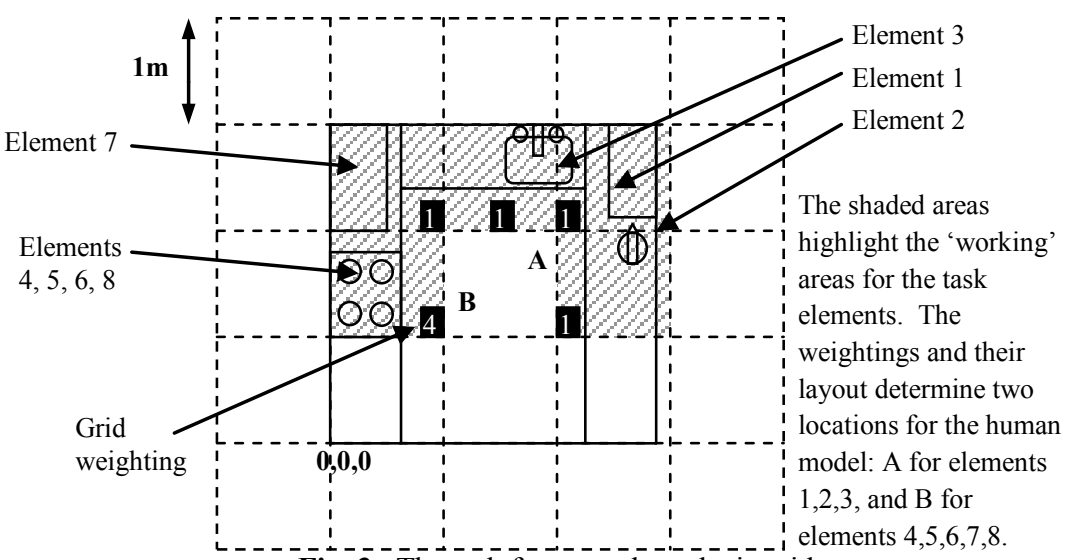

Fig. 3. The task framework analysis grid.

\subsection{Human Models}

The human models of the system are visual representations of the user population. In addition they are data sets that reflect the variety within the population of characteristics such as anthropometry, somatotype, capability, handedness and behaviour. Thus the human models are stored as individuals and their embedded data will be used to influence how they address the task.

The behavioural details are a critical element in how the system synthesises a task element posture when the default approach is not sufficient. As an example, a task element requires an individual to place an object in an oven. The first check is to see if that person could reach the appropriate place in the oven from a normal (default) posture. Assuming the test to be a failure the question then asked is what postural changes are made to ensure a success? Whilst a strategy has been established in Section 2.4.2, a general approach cannot be adequate when dealing with the variety of human behaviour. Thus, each individual human model contains data on their behaviour to common task elements such as reaching below the level of the hips (do they bend their back, do they bend their legs, do they crouch, do they sit on the floor?). This information then modifies the generic process shown in Section 2.4.2 to an appropriate response for that individual. 


\section{Conclusions and Further Work}

To support those endeavouring to design for all, a tool has been developed called HADRIAN that works in conjunction with an existing human modelling tool, SAMMIE. HADRIAN provides an accessible and applicable database of 100 individuals. These data can then be employed in a task analysis system that simplifies and automates the analysis performed in a typical human modelling system. In addition, the analysis is enhanced through a series of mechanisms that attempt to replicate a real task being performed by real people in a virtual task simulation. The underlying methodology employed by the task analysis system is an ongoing area of research and its implementation is currently undergoing validation in two studies to examine its performance against both the use of a human modelling system by an expert, and the benchmark of a real user trial. It is intended that the validation findings will be used to refine and improve the HADRIAN model outlined in this paper.

\section{References}

1. Marshall, R., Summerskill, S.J., Porter, J.M., Case, K., Sims, R.E., Gyi, D.E. and Davis, P.M., 2008. Multivariate design inclusion using HADRIAN, Proceedings of the SAE 2008 Digital Human Modelling for Design and Engineering Conference and Exhibition, SAE Paper No 2008-01-1899, Pittsburgh, USA, June 2008, pp 1-8.

2. Adultdata., 1998. The handbook of adult anthropometry and strength measurements - data for design safety. L. Peebles and B. Norris, eds. Department of Trade and Industry.

3. Treleaven, P., 2007. How to fit into your clothes: Busts, waists, hips and the UK National Sizing Survey. Significance, 4 (3), 113-117.

4. CAESAR., 2008. Civilian American and European Surface Anthropometry Resource [online]. Available from: http://store.sae.org/caesar/ [Accessed 26/02/2009].

5. Paquet, V. and Feathers, D., 2004. An anthropometric study of manual and powered wheelchair users, International Journal of Industrial Ergonomics, 33 (3), 191-204.

6. Das, B. and Kozey, J., 1999. Structural anthropometric measurements for wheelchair mobile adults. Applied Ergonomics 30 (5), 385-390.

7. Gyi, D.E., Sims, R.E., Porter, J.M., Marshall, R. and Case, K., 2004. Representing older and disabled people in virtual user trials: data collection methods", Applied Ergonomics, 35 (5), 443-451.

8. Porter, J.M., Case, K., Marshall, R., Gyi, D.E. and Sims, R.E., 2004. Beyond Jack and Jill: designing for individuals using HADRIAN, International Journal of Industrial Ergonomics, 333,1st March 2004, 249-264.

9. Marshall, R., Case, K., Porter, J.M., Sims, R.E. and Gyi, D.E., 2004. Using HADRIAN for Eliciting Virtual User Feedback in 'Design for All', Journal of Engineering Manufacture; Proceedings of the Institution of Mechanical Engineers, Part B, 218(9), 1st September 2004, 1203-1210.

10.Porter, J.M., Marshall, R., Freer, M. and Case, K., 2004. SAMMIE: a computer aided ergonomics design tool. In: N.J. Delleman, C.M. Haslegrave, and D.B. Chaffin eds. Working Postures and Movements - tools for evaluation and engineering. Boca Raton: CRC Press LLC, 454-462. 